\title{
REAL TIME IMPLEMENTATION OF OBJECT TRACKING THROUGH
}

\section{WEBCAM}

\author{
Sherin Cherian ${ }^{1}$, C.Senthil Singh ${ }^{2}$ \\ ${ }^{1}$ M.Tech Student, ${ }^{2}$ Associate Professor, Electronics and Communication Engineering, Toc H Institute of Science and \\ Technology, Kerala, India
}

\begin{abstract}
Real time object detection and tracking is an important task in various computer vision applications. For robust object tracking the factors like object shape variation, partial and full occlusion, scene illumination variation will create significant problems. We introduce object detection and tracking approach that combines Prewitt edge detection and kalman filter. The target object's representation and the location prediction are the two major aspects for object tracking this can be achieved by using these algorithms. Here real time object tracking is developed through webcam. Experiments show that our tracking algorithm can track moving object efficiently under object deformation, occlusion and can track multiple objects.
\end{abstract}

Keywords: Object Tracking, real time, kalman filter, Prewitt edge detection.

\section{INTRODUCTION}

Object Tracking can be defined as the problem of finding the location of an object in the image plane when it moves its position from frame to frame. In other words a tracker assigns appropriate labels to the tracked objects in different frames associated with the video that we captured. While considering a single image it provides a snapshot of a scene, where as in video that we capture over certain time represents the dynamics in the scene. Tracking objects can be complex due to variation in object structures, loss of information caused by projection of the $3 \mathrm{D}$ world on a $2 \mathrm{D}$ image, real-time processing requirements, complex object motion etc. There are mainly three steps associated with tracking and they are, detecting the moving objects, tracking the detected objects from frame to frame and analyze the behaviour of tracked object. In tracking almost all algorithms assume that the object motion is smooth with no abrupt changes we need a prior knowledge about the number and size of object, shape and appearance of the object.

In a tracking scenario, whatever may be an object it can be a boat on a sea, vehicle on a road, plane in an air or people walking on a road. Generally, there is a strong relationship between the object representations and the tracking algorithms [1]. Object representation can be chosen on the basis of application and object can be represented on the basis of shape and appearance. In object tracking selecting the right feature plays a critical role. The most desirable property of a visual feature is its uniqueness that the objects can be easily distinguished in the feature space. There is a close relationship between object representation and tracking. For example, in the case of contour-based representation we choose object edges as a feature and for histogram based representation we consider colour as a feature. In most of the cases the tracking algorithm considers a combination of features.

It is important to develop a real time working algorithm though it is a challenging task. In recent years real-time working systems [7]-[12] and a number of tracking algorithms [1]-[6] have been developed. Particle filter [2],[6],[8],[13] is a powerful method to localize target, it gives high-precision results in complex situations, where as it is computationally expensive so particle filter is not suitable for real time Implementation and it is highly sensitive to noise too. But particle filter is good for target object representation and location prediction. Certain works proposed various improvements over particle filter for better tracking under complex situations [2]. It is based on a Spatial-colour Mixture of Gaussians (SMOG) appearance model for particle filters. This method improves the similarity measure based on colour histograms because it considers not only the colours in a region but also the spatial layout of the colours. It is a powerful method for localizing the target and provides high precision result even under complex situation. Because of high computational cost which prevents their implementation from working in real time.

In most of the tracking algorithm, the tracking performance depends up on the target image representation. Colour, texture and edge are typical attributes used for representing objects [20], [21] beyond this SIFT feature [22], optical flow [23], orientation energy[5] and active contours[11] are also used in many of the works. [25] it was revealed that the visual perception of animals relies heavily on the directional edges so that they are having excellent ability in visual tracking. By employing the directional-edge-based feature vector 
representation, the system has been made robust against illumination variation and small variation in object shapes. To realize the tracking function, they use multiple candidate regeneration and online learning is adopted to improve the tracking performance. Meanshift Algorithm can also be used for tracking [20], here they consider adaptive colour model for MeanShift tracking. The paper consists of two major aspects. The object colour is analyzed based on a clustering algorithm and according to the clustering result, the colour space of the object is partitioned into subspaces by orthonormal transformation. This algorithm is having good tracking performance when compared with conventional MS algorithm. But they suffer from the high computational cost.

For estimating the position of moving object [24] consider Kalman filter as an estimator. Here kalman filter has been used successfully in different prediction applications. Where the input is considered as a sequence of images captured by a camera that containing the object. There are mainly 3 steps associated with kalman filter for visual tracking and they are Initialization, Prediction and finally Correction.

The purpose of this paper is to develop a real time object tracking by using edge as a feature which is robust against some complex situations like scene illumination variation, object shape deformation, full and partial occlusion of moving object. In this paper object tracking is achieved by taking the real time video sequence from webcam. prewit operator is used for object detection, after detecting the edge of the object tracking is achieved through kalman filter.

\section{METHODOLOGY}

In this section, we discuss the methodology we followed for the process of real time object tracking by object detection and tracking. Prewit operator is used for detecting the edge of the captured video sequence from the webcam and tracking is achieved through kalman filter.

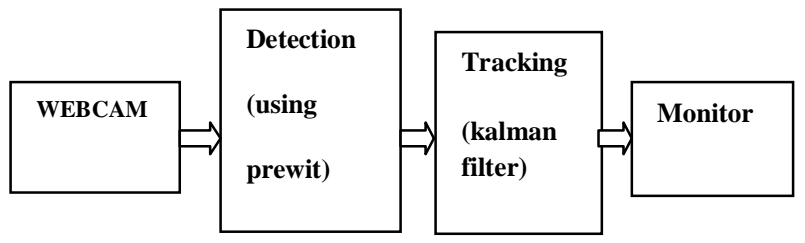

Fig -1: Block Diagram

\subsection{Prewit Edge Detection}

Prewitt operator is an edge detection method which mainly detects two types of edges in an image:

- Horizontal edges

- Vertical Edges
Edges are calculated on the basis of the difference between corresponding pixel intensities of an image. The two masks that are used for edge detection are known as the derivative masks. The reason for considering differentiation is that since image is so changes in a signal can only be calculated using differentiation. This is the reason why these operators are also called as derivative masks or derivative operators.

The following points show the properties of derivative mask:

- $\quad$ Each mask should contain opposite sign.

- Sum of each mask should be equal to zero.

- More weight means implies more edge detection.

Let us consider $\mathrm{f}(\mathrm{x}, \mathrm{y})$ is the image gray scale distribution and $\mathrm{P}(\mathrm{x}, \mathrm{y})$ is the edge of the image gradient value, Gx and Gy are the images which at each point contains the horizontal and vertical derivative. Prewitt operator provides 2 masks one for detecting edges in horizontal direction and another for detecting edges in an vertical direction. The values associated with Gx and Gy are

\begin{tabular}{|l|l|l|}
\hline-1 & 0 & 1 \\
\hline-1 & 0 & 1 \\
\hline-1 & 0 & 1 \\
\hline
\end{tabular}

\begin{tabular}{|l|l|l|}
\hline 1 & 1 & 1 \\
\hline 0 & 0 & 0 \\
\hline-1 & -1 & -1 \\
\hline
\end{tabular}

Here $P(x, y)=\sqrt{ }(G x 2+G y 2$. Appropriate magnitude can be computed using $|\mathrm{G}|=|\mathrm{Gx}|+|\mathrm{Gy}|$ and the angle of orientation of the edge is $\Theta=\arctan (\mathrm{Gx} / \mathrm{Gy})-(3 \pi / 4)$. Where $*$ denotes the 2-dimensional convolution operation. Prewit operator uses partial differential method for edge detection method to find the edge of the operator

In the case of vertical direction, the above mask the zeros column in the vertical portion is used to find the edges in the vertical direction. After convolving this mask on an image it gives you the vertical edges in an image. It is a partial differentiation method used to calculate the difference of pixel intensities in a edge region. Here the centre column contains value as zero so it does not include the original values of an image but it calculates the difference of right and left pixel values around that edge. Thus edge intensity is increased and it becomes enhanced comparatively to the original image.

In the case of horizontal direction, the above mask the zeros column in the horizontal portion is used to find the edges in the horizontal direction. After convolving this mask on an image it gives you the horizontal edges in an image. This also works on the principle of above mask and it calculates difference among the pixel intensities of a particular edge. The canter row of mask consist of zeros so it does not include the original values of edge in the image but it calculates the difference of above and below pixel intensities of the particular edge. Increasing the sudden change of intensities and making the edge more visible. 


\subsection{Kalman Filter}

The Kalman filter possesses many applications in technology. It is an essential part of both military technology and the development of space technology. The main function of Kalman Filter is to utilize measurements recorded over time which contain random variations and inaccuracies to generate values that tend closer to the measurement's true values and connected values that resulted from calculations. For estimating the position of object is performed using the Kalman filter as an estimator. It has been used successfully in different prediction applications or state determination of a system. Kalman filter as a probabilistic prediction method for object tracking. Main problems that can be solved by using kalman filter in tracking is

- The object can be track if it move beyond the searched region

- Variation factors such as lighting and occlusion which effect the appearance of target.

The Kalman filter is a framework for predicting a process's state, and using measurements to correct or 'update' these predictions. The Kalman filter estimates a process by using a form of feedback control which means the filter estimates the process state at some time and then obtains feedback in the form of (noisy) measurements. The equations associated with Kalman filter fall into two groups: time update equations and measurement update equations.

The time update equations point towards the current state estimate and error covariance prediction is used to obtain the main estimates for the next time step. Then measurement update step is responsible for the feedback by calculating the kalman gain. The time update equations is associated with prediction, while the measurement update equations is associated with correction equations. Kalman filter is a recursive solution to the discrete-data linear filtering problem. The Kalman filter is essentially a set of mathematical equations that implement a predictor-corrector type estimator that is optimal in the sense that it minimizes the estimated error covariance when some presumed conditions are met. The Kalman filter has been used extensively for tracking in interactive computer graphics.

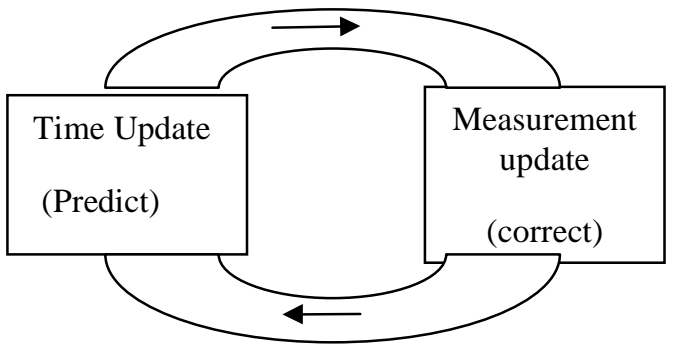

Fig 2: Kalman Filter

\subsubsection{Time Update}

In the case of kalman filters each iteration begins with predicting the process's state using a linear dynamics model.

1. State Prediction: For each time step T, a Kalman filter first makes a prediction $X_{t}$ and it is given by

$$
\mathbf{X}_{t}=\mathbf{A} \mathbf{x}_{\mathbf{t}-1}+\mathbf{B} \mathbf{u}_{\mathbf{t}}
$$

$\mathrm{x}_{\mathrm{k}-1}$ is process state at time $\mathrm{t}-1, \mathrm{~A}$ is process transition matrix and $B$ is control vector. $U_{t}$ which converts control vector into state space

In our model of moving objects on webcam images, state is a 4-dimensional vector $[\mathrm{x} ; \mathrm{y} ; \mathrm{dx} ; \mathrm{dy}$ ] where $\mathrm{x}$ and $\mathrm{y}$ shows the coordinates of the object's centre and $\mathrm{dx}$ and dy shows its velocity.

2. Error Covariance Prediction: The Kalman filter concludes the time update steps by estimating the error covariance forward by one time step

$$
\mathbf{P}_{\mathbf{t}}=\mathbf{A} \mathbf{P}_{\mathbf{t}-1} \mathbf{A}^{T}+\mathbf{Q}
$$

$\mathrm{P}_{\mathrm{t}-1}$ is a matrix representing error covariance in the state prediction at time $\mathrm{t}-1$ and $\mathrm{Q}$ is the process noise covariance. Lower the value of prediction error covariance $P_{t}$ we can trust more on prediction of the state $\mathrm{x}_{\mathrm{t}}$. If the process is precisely modeled, then the prediction error covariance will be low.

\subsubsection{Measurement Update}

By using the time update step kalman filter will predict the state $\mathbf{x}_{\mathbf{t}-\mathbf{1}}$ and find the error covariance at time $\mathrm{k}$. then after during the measurement update steps kalman filter uses measurements to correct its prediction

\section{Kalman Gain}

Kalman filter computes a Kalman gain $\mathrm{K}_{\mathrm{t}}$, which is used for correcting the state estimate $\mathrm{x}_{\mathrm{t}}$

$$
\mathbf{K}_{\mathrm{k}=} \mathbf{P}_{\mathrm{k}} \mathbf{H}^{T}\left(\mathbf{H} \mathbf{P}_{\mathrm{k}} \mathbf{H}^{T}+\mathbf{R}_{\mathrm{k}}\right)^{\wedge}-1
$$

Where $\mathrm{H}$ is a matrix used for converting into measurement space from state space and $\mathrm{R}$ is measurement noise covariance.

2. State Update: Using Kalman gain $K_{t}$ and measurements $Z_{t}$ from time step $\mathrm{t}$, where we can update the state estimate:

$$
\mathbf{X}_{\mathbf{t}}=\mathbf{X}_{\mathrm{t}-1}+\mathbf{X}_{\mathbf{t}}\left(\mathbf{Z}_{\mathrm{t}}-\mathrm{H} \mathbf{X}_{\mathrm{t}-1}\right)
$$

3. Error Covariance Update: The final step of the Kalman filter's iteration is to update the error covariance $\mathrm{P}_{\mathrm{t}}$.

$$
\mathbf{P}_{\mathbf{t}}=\left(1-\mathbf{K}_{t} \mathrm{H}\right) \mathbf{P}_{\mathbf{t}-1}
$$


If the measurements are accurate then the updated error covariance will be decreased.

\section{EXPERIMENTAL RESULT}

A series of experiments were conducted to finding the tracking accuracy of our method. We evaluated the performance of our tracking method by using real time video which is taken directly from the camera.

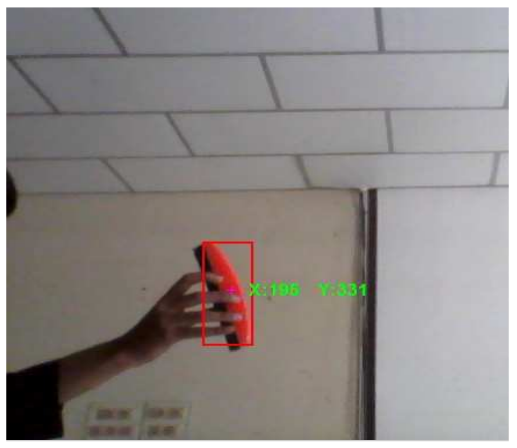

Fig 3: Tracking a varied shape object

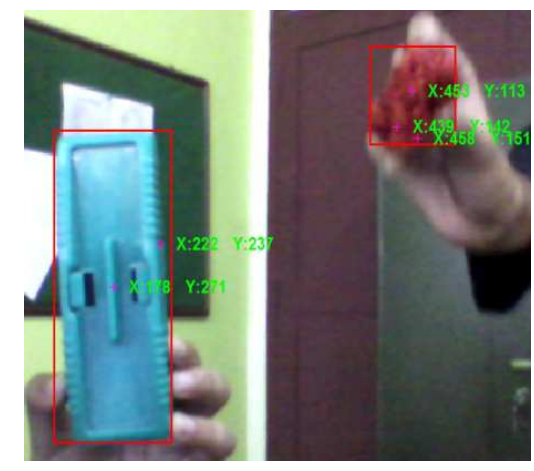

Fig 4: Multiple tracking

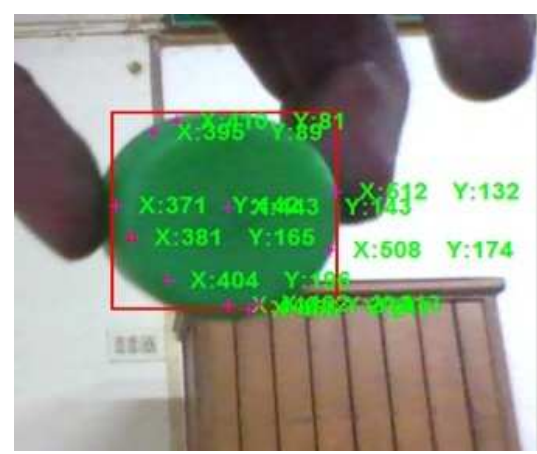

Fig5: Tracking of a round object

Here the position of moving object is also marked ie, its $\mathrm{X}$ and $\mathrm{Y}$ positions. Here the frame rate is $30 \mathrm{fps}$.
Fig 3 shows the tracking of a varied shape object. It shows here tracking is unaffected with complex shape variation. Fig 4 shows multiple tracking is made possible and Fig 5 shows the tracking of round object

\section{CONCLUSIONS}

In this work, the combined algorithm is used for extracting moving objects from a real time video stream is obtained from webcam. For tracking and detection purpose kalman filter and prewit edge detection is used. These algorithms are suitable for improving the performance by target object's representation and predicting the location. In the present work moving object can be effectively tracked under complex situations like full occlusion, object shape variation and can achieve multiple object tracking.

\section{REFERENCES}

[1] A. Yilmaz, O. Javed, and M. Shah, "Object tracking: A survey," ACM Computing Surveys, vol. 38, no. 4, 2006.

[2] H. Wang, D. Suter, K. Schindler, and C. Shen, "Adaptive object tracking based on an effective appearance filter," Pattern Analysis and Machine Intelligence, IEEE Transactions on, vol. 29, no. 9, pp. $1661-1667$, sept. 2007.

[3] B. Han, Y. Zhu, D. Comaniciu, and L. Davis, "Visual tracking by continuous density propagation in sequential Bayesian filtering framework," Pattern Analysis and Machine Intelligence, IEEE Transactions on, vol. 31, no. 5, pp. 919 -930, may 2009.

[4] Y.-J. Yeh and C.-T. Hsu, "Online selection of tracking features using adaboost," Circuits and Systems for Video Technology, IEEE Transactions on, vol. 19, no. 3, pp. 442 -446, march 2009.

[5] Q. Chen, Q.-S. Sun, P. A. Heng, and D.-S. Xia, "Twostage object tracking method based on kernel and active contour," Circuits and Systems for Video Technology, IEEE Transactions on, vol. 20, no. 4, pp. $605-609$, april 2010.

[6] Z. Khan, I. Gu, and A. Backhouse, "Robust visual object tracking using multi-mode anisotropic mean shift and particle filters," Circuits and Systems for Video Technology, IEEE Transactions on, vol. 21, no. 1, pp. $74-87$, jan. 2011.

[7] J. U. Cho, S. H. Jin, X. D. Pham, J. W. Jeon, J. E. Byun, and H. Kang, "A real-time object tracking system using a particle filter," in Intelligent Robots and Systems, 2006 IEEE/RSJ International Conference on, oct. 2006, pp. $2822-2827$.

[8] H. Medeiros, J. Park, and A. Kak, "A parallel colorbased particle filter for object tracking," in Computer Vision and Pattern Recognition Workshops, 2008. CVPRW '08. IEEE Computer Society Conference on, june 2008, pp. $1-8$. 
[9] Z. Kim, "Real time object tracking based on dynamic feature grouping with background subtraction," in Computer Vision and Pattern Recognition, 2008. CVPR 2008. IEEE Conference on, june 2008, pp. 1 -8.

[10] T. Ishiguro and R. Miyamoto, "An efficient prediction scheme for pedestrian tracking with cascade particle filter and its implementation on cell/b.e." in Intelligent Signal Processing and Communication Systems, 2009. ISPACS 2009. International Symposium on, jan. 2009, pp. $29-32$.

[11] E. Norouznezhad, A. Bigdeli, A. Postula, and B. Lovell, "Robust object tracking using local oriented energy features and its hardware/software implementation," in Control Automation Robotics Vision (ICARCV), 2010 11th International Conference on, dec. 2010, pp. $2060-2066$.

[12] S.-A. Li, C.-C. Hsu, W.-L. Lin, and J.-P. Wang, "Hardware/software codesign of particle filter and its application in object tracking," in System Science and Engineering (ICSSE), 2011 International Conference on, june 2011, pp. $87-91$.

[13] A. Doucet, On sequential simulation-based methods for Bayesian filtering. Tech. Rep., Cambridge Univ., 1998.

[14] H. Medeiros, X. Gao, J. Park, R. Kleihorst, and A. Kak, "A parallel implementation of the color-based particle filter for object tracking," in Proceedings of the ACM Sensys Workshop on Applications, Systems and Algorithms for Image Sensing (ImageSense'08). Citeseer, 2008.

[15] D. Cherng, S. Yang, C. Shen, and Y. Lu, "Real time color based particle filtering for object tracking with dual cache architecture," in Advanced Video and Signal-Based Surveillance (AVSS), 2011 8th IEEE International Conference on. IEEE, 2011, pp. 148-153.

[16] X. Lu, D. Ren, and S. Yu, "Fpga-based real-time object tracking for mobile robot," in Audio Language and Image Processing (ICALIP), 2010 International Conference on. IEEE, 2010, pp. 1657-1662.

[17] S. Liu, A. Papakonstantinou, H. Wang, and D. Chen, "Real-time object tracking system on fpgas," in Application Accelerators in High- Performance Computing (SAAHPC), 2011 Symposium on. IEEE, 2011, pp. 1-7.

[18] Y.-M. Lin, C.-H. Yeh, S.-H. Yen, C.-H. Ma, P.-Y. Chen, and C.-C. Kuo, "Efficient VLSI design for SIFT feature description," Next-Generation Electronics (ISNE), 2010 International Symposium on, pp. 48-51, 2010.

[19] H. El, I. Halym, and S. ed Habib, "Proposed hardware architectures of particle filter for object tracking," EURASIP Journal on Advances in Signal Processing, vol. 2012, no. 1, p. 17, 2012.

[20] P. Li, "An adaptive binning color model for mean shift tracking," Circuits and Systems for Video Technology, IEEE Transactions on, vol. 18, no. 9, pp. $1293-1299$, sept. 2008.
[21] J. Wang and Y. Yagi, "Integrating color and shapetexture features for adaptive real-time object tracking," Image Processing, IEEE Transactions on, vol. 17, no. 2, pp. $235-240$, feb. 2008.

[22] S. Fazli, H. Pour, and H. Bouzari, "Particle filter based object tracking with sift and color feature," in Machine Vision, 2009. ICMV '09. Second International Conference on, dec. 2009, pp. 89 -93.

[23] S. Avidan, "Support vector tracking," Pattern Analysis and Machine Intelligence, IEEE Transactions on, vol. 26, no. 8, pp. $1064-1072$, aug. 2004.

[24] M.Farrokhi, B.Torkaman, "A Kalman-Filter-Based Method for Real-Time Visual Tracking of a Moving Object Using Pan and Tilt Platform," International Journal of Scientific \& Engineering Research Volume 3, Issue 8, August-2012

[25] He Li, Hongbo Zhu, Pushe Zhao, and Tadashi Shibata," A Directional-Edge-Based Real-Time Object Tracking System Employing Multiple CandidateLocation Generation" 2013, IEEE

\section{BIOGRAPHIES}

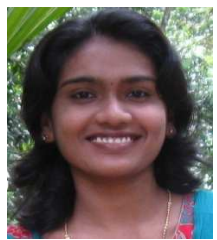

Sherin Cherian received the B.Tech degree in Electronics and Communication Engineering from Mahatma Gandhi University, India, in 2012.She is doing her M.Tech in VLSI and Embedded System from Cochin University of Science and Technology, India. Her area of interest includes VLSI, Video Processing

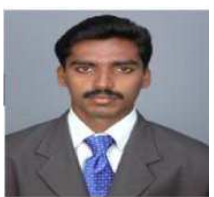

C.Senthil singh received the B.E. degree in Electrical and Electronics Engineering from the MS University, India, MTech in VLSI Design, Currently doing Ph.D. in Information and Communication in Anna University, Chennai, India. His research interest includes VLSI, Image processing. 